\title{
Promoting Dietary Behavior Using the Mediterranean Diet in an Online College Environment
}

\author{
Basil H. Aboul-Enein ${ }^{1}$ and Joshua Bernstein ${ }^{2}$ \\ ${ }^{1}$ University of South Dakota \\ ${ }^{2}$ A.T. Still University
}

\begin{abstract}
Background and Purpose: The prevalence of obesity has prompted calls for broad public health education solutions to reduce, if not reverse, overweight and obesity among college students in the United States. This study investigated change in student dietary behavior and food choices following an education intervention that introduced the Mediterranean diet (MD) as a weight management tool in a Houston, Texas community college. Methods: Online survey data were collected at pre-test and post-test assessments from a convenience sample of 65 college students enrolled in an online undergraduate nutrition course during the summer of 2013. The Mediterranean Diet Quality Index (KIDMED) survey evaluated dietary behavior change based on the MD Principals. Results: The intervention improved total participant population from a mean KIDMED score of poor (4.12) to a mean score of high (8.45), indicating improved dietary behavior change, particularly among men. Significant differences in KIDMED change scores by ethnicity and heart disease (HD) history were also observed. Conclusion: This study provides a unique pedagogical illustration of e-learning to promote MD patterns among college student populations.
\end{abstract}

(C) 2014 Californian Journal of Health Promotion. All rights reserved.

Keywords: Nutrition, overweight, obesity, college students, Mediterranean diet

\section{Introduction and Purpose}

\section{College Obesity}

Many U.S. college students increase their risk of developing heart disease (HD) due to becoming overweight or obese from unhealthy lifestyle behaviors (Fernandes, Arts, Dimond, Hirshberg, \& Lofgren, 2013). A national survey by the American College Health Association (ACHA) (Sparling, 2007) in 2005 found three of 10 college students were either overweight (BMI between 25-29.9) or obese (BMI between 3039.9), and nine of 10 students consumed fewer than five servings of fruits and vegetables daily. The consumption of calorically dense convenience foods are related to overweight and obesity, and they have displaced nutritious options, particularly fresh fruits and vegetables (United States Department of Health and Human Services, 2005). Other factors contributing towards excessive caloric intake include lowcost, energy-dense foods, increased consumption of processed meals and sugary drinks, and ample opportunities to eat throughout the day (Brunt \& Rhee, 2008). Dietary recommendations to reduce obesity advocate a diet rich in fruits, vegetables, and whole grains. A modern nutrient dense guideline that advocates this pattern is the Mediterranean diet (MD) (Tourlouki et al., 2010).

The MD has been characterized by an abundance of plant-based foods, whole grain cereals, low to moderate consumptions of dairy products, liberal use of olive oil, low to moderate consumptions of fish and poultry, rare consumption of red meat, and moderate amounts of wine consumed with meals (Tourlouki et al., 2010). Findings indicate MD adherents experience some of the lowest rates of chronic disease in the world, some of the highest life expectancies in the world, and protective effects against obesity-related co-morbidities such as diabetes and HD (Tourlouki et al., 2010). 


\section{Online-based Intervention Promoting the Mediterranean Diet}

The number of students taking online courses is increasing. From 2002 to 2011, the number of online U.S. students increased from 16.6 million to 21 million. This represents a compound annual growth rate of $17.3 \%$ of students taking online courses, compared to a $2.6 \%$ growth rate for higher education students in general. With $34 \%$ of U.S. college students now enrolled in at least one online course, the highest rate on record (Allen \& Seaman, 2013), recent studies suggest colleges and universities are ideal settings for overweight and obesity prevention, using an online educational intervention versus a traditional, destination-based service where students seek out health information. Considering that very few studies (e.g. Papadaki \& Scott, 2005) have examined the effectiveness of the MD as a web-based health intervention for nutrition education or in a college environment, this study used an online course management system to expose college students to MD patterns.

\section{The Present Study}

The purpose of this study was to implement and evaluate an online nutrition module using the $\mathrm{MD}$, and measure behavior change among college students with consideration for sex, ethnicity, and family history of HD. The research questions answered in this study included:

1. Is there statistically significant difference between pre-test and post-test behavior scores following a web-based health education module?

2. Does behavior change from pre-test to post-test vary according to gender, ethnicity, and student family history of HD?

\section{Methods}

\section{Design and Sample}

This study used a single cohort pretest-posttest design. Expedited Institutional Review Board (IRB) approval was secured from San Jacinto College and A.T. Still University of Health Sciences. An educational intervention based on the MD was created as a module in an online nutrition and diet therapy course, made available to all 80 online undergraduate students enrolled in the course. The module introduced the MD, in both historical and contemporary contexts, as a potential weight management guideline. The module included evidence-based peer-reviewed reports and published articles that examined the MD (Huang \& Sumpio, 2008; Issa et al., 2011). Educational tools included guided presentations, literature exploration, and demonstration videos; assessment tools included interactive discussion forums, recipe analyses, research projects, and a dietary questionnaire. Responses to the questionnaire were analyzed in this study.

Of the 80 students eligible to participate in the study because of their enrollment in the course, 65 students (75\%) provided consent to participate in the study. Participants were free to decline participation or opt out of the study at any time without penalty or prejudice. The anonymous questionnaire was posted in the online course platform, along with a statement of potential risk and informed consent reviewed and accepted by all participants.

\section{Measures}

Data was collected using the KIDMED index, a 16-item questionnaire, which traditionally classifies participant behaviors (adherence and compliance to the MD) as: $\leq 3$ (low), 4-7 (medium), and $\geq 8$ (high) (Serra-Majem, Ribas, Garcia, Perez-Rodrigo, \& Aranceta, 2003). The KIDMED index ranges from 0 to 12 and includes 12 positive questions and 4 negative questions. Each answers is given a value of +1 or 0 (for positive behaviors) or -1 or 0 (for negative behaviors), and responses to all items are summed. Sahingoz and Sanlier (2011) validated the KIDMED index to assess adherence and compliance $(\alpha \geq 0.84)$.

\section{Analyses}

Data was analyzed using SAS Enterprise Guide 4.3. A one-way ANOVA and Tukey post-hoc test for significance compared pre-test participant KIDMED data by sex, ethnicity, and family HD. The posttest minus pretest KIDMED difference scores were also analyzed for normality and further descriptive statistics were provided (graphical mean and standard 
deviation). Difference scores were compared by gender, ethnicity (Caucasian vs. non-Caucasian), and HD status (family history vs. no family history of heart disease) by calculating three one-way ANOVAs, one for each demographic variable.

\section{Results}

Differences by Gender Pretest score analysis revealed higher KIDMED scores among females relative to males $(p<0.001)$. Although females $(\bar{x}=8.68, \pm 2.12$, high) scored higher than males $(\bar{x}=7.67, \pm 2.06$, medium) in the posttest, their scores were no longer statistically different from each other. $(p=0.108)$. When difference scores were compared, males demonstrated significantly greater improvement in KIDMED scores compared to females ( $\mathrm{F}=2.66, p<0.001$, $\alpha=0.05)$. See Table 1 .

Table 1

KIDMED Score Comparison by Sex

\begin{tabular}{lrrr}
\hline Sex & $\begin{array}{l}\text { Pre-test } \boldsymbol{M} \\
\text { (SD) }\end{array}$ & $\begin{array}{l}\text { Post-test } \\
\boldsymbol{M} \text { (SD) }\end{array}$ & \multicolumn{1}{l}{$\begin{array}{l}\text { Pre/Post } \boldsymbol{M} \\
\text { Difference(SD) }\end{array}$} \\
\hline Female $(n=50)$ & $4.86(2.81)$ & $8.68(2.12)$ & $+3.82(2.00)$ \\
Male $(n=15)$ & $1.67(2.16)$ & $7.67(2.06)$ & $+6.00(2.00)$ \\
$p$-value & 0.001 & 0.108 & 0.005 \\
\hline
\end{tabular}

\section{Differences by Ethnicity}

Results indicated differences $(p<0.004)$ between pretest Caucasian scores $(\bar{x}=2.81, \pm$ 3.03 , poor $)$ and non-Caucasian scores $(\bar{x}=5.32$, \pm 2.40 , medium). Non-Caucasians overall scored higher than Caucasians in the posttest, both ethnicity scores fell within the same categorical group (high), and score differences between groups failed to reach statistical significance ( $p$ $=0.171)$. When difference scores were compared, Caucasians demonstrated greater improvements in KIDMED scores compared to non-Caucasians ( $\mathrm{F}=1.92, p<0.001, \alpha=0.05)$. See Table 2.

\section{Differences by HD History}

Pretest score analysis revealed the non-HD group had higher KIDMED scores than the group with HD history $(p<0.001)$. The mean posttest score for the non-HD history group was $8.60( \pm 2.30$, high $)$ and the HD history group was $8.30( \pm 1.80$, high); these two means were not statistically different from each other. When difference scores were compared, those with a family history of heart disease demonstrated greater improvements in KIDMED scores ( $\mathrm{F}=$ 1.04, $p<0.001, \alpha=0.05)$. Please see Table 3.

Table 2

KIDMED Score Comparison by Ethnicity

\begin{tabular}{lrrr}
\hline Ethnicity & \multicolumn{1}{l}{$\begin{array}{l}\text { Pre-test } \boldsymbol{M} \\
\text { (SD) }\end{array}$} & $\begin{array}{l}\text { Post-test } \\
\boldsymbol{M} \text { (SD) }\end{array}$ & $\begin{array}{l}\text { Pre/Post } \boldsymbol{M} \\
\text { Difference (SD) }\end{array}$ \\
\hline Caucasian $(n=31)$ & $2.81(3.03)$ & $8.06(2.30)$ & $+5.25(2.08)$ \\
$\begin{array}{l}\text { Non-Caucasian } \\
(n=34)\end{array}$ & $5.32(2.40)$ & $8.79(2.03)$ & $+3.47(1.96)$ \\
$p$-value & & & \\
\hline
\end{tabular}

Table 3

KIDMED Score Comparison by Family History of HD

\begin{tabular}{lrrr}
\hline HD History & \multicolumn{1}{l}{$\begin{array}{l}\text { Pre-test } \boldsymbol{M} \\
\text { (SD) }\end{array}$} & \multicolumn{1}{l}{$\begin{array}{l}\text { Post-test } \\
\boldsymbol{M} \text { (SD) }\end{array}$} & \multicolumn{1}{l}{$\begin{array}{l}\text { Pre/Post } \boldsymbol{M} \\
\text { Difference (SD) }\end{array}$} \\
\hline No $(n=40)$ & $5.00(3.00)$ & $8.60(2.30)$ & $+3.60(2.02)$ \\
Yes $(n=25)$ & $2.72(2.41)$ & $8.30(1.80)$ & $+5.58(1.89)$ \\
$p$-value & 0.021 & 0.624 & 0.002 \\
\hline
\end{tabular}

\section{Discussion}

This study designed, implemented, and evaluated a health promotion intervention that addressed behaviors associated with overweight, obesity, and HD history among college students. The online pedagogical approach is relatively unique; traditional student health and wellness programs are standard and expected for undergraduate students, while equivalent online programs are comparatively uncommon (Thompson \& Porto, 2014, Hermon \& Davis, 2004). Due to the significant increase in popularity of online college courses and programs (Allen \& Seaman, 2013), this methodological design represents a useful, relatively inexpensive, and popular avenue to engage online college students.

Total participant population improved from a mean KIDMED score of poor to a mean score of high suggesting the intervention may have influenced dietary behavior change. Across the entire participant sample, males, Caucasians, and participants with a family history of HD benefited from the intervention more significantly. A possible explanation for this 
finding is the existing dietary habits of nonCaucasian females may be more in line with the MD based on cultural preferences, as indicated by the significantly higher pre-test scores and the relatively small improvement pretest to posttest. Family history of HD participants may feel particularly susceptible to HD when cued or reminded of their potential risk for HD. The HD group might also be acutely affected by this intervention as evidenced by a significant behavior shift (low to high) and view the MD as a HD risk reduction tool in addition to a weight control tool. Our findings specific to Caucasians and non-Caucasians may indicate web-based health education approaches using MD guidelines could effectively target either an ethnically diverse or an ethnically homogenous college student population.

\section{Limitations}

Our limited sample size did not allow us to include sex, ethnicity, and family history into one model predicting change in KIDMED scores. Hence it is not clear which demographic variable is most strongly related to changes in KIDMED scores. Lack of an experimental control group limited our ability to determine the effect of the MD intervention course relative to a standard nutrition course. Also, participants may have responded in a socially desirable fashion when surveyed about dietary behavior, such that KIDMED scores were higher than the behaviors they represent. Finally, this study was conducted in one Texas community college and included only one online course of students. These study limitations limit the generalizability of our study findings to colleges and universities in other geographic regions.

\section{Conclusions}

This study provides health educators with a unique pedagogical illustration of e-learning to promote an alternative nutritional guideline to address overweight and obesity. Findings suggest future research focused on sex-tailored nutrition interventions, nutrition interventions targeting individuals with family history of HD, and multiple group pretest-posttest study designs among college student populations are warranted.

\section{Acknowledgments}

The authors would like to thank Dr. James Baker, Dr. Casey Durand, and Dr. Larry Olsen for their editing assistance and the nutrition students at San Jacinto College who participated in the study. The authors would also like to thank Dr. Robert Wills and Dr. Jacquelyn Bowser for their assistance with data analysis.

\section{References}

Allen, I. E., \& Seaman, J. (2013). Changing course: Ten years of tracking online education in the United States. Upper Saddle River, NJ: Pearson.

Bargiota, A., Pelekanou, M., Tsitouras, A., \& Koukoulis, G. N. (2013). Eating habits and factors affecting food choice of adolescents living in rural areas. Hormones, 12, 246-53.

Fernandes, J., Arts, J., Dimond, E., Hirshberg, S., \& Lofgren, I. E. (2013). Dietary factors are associated with coronary heart disease risk factors in college students. Nutrition Research, 33, 647-52.

Hermon, D. A., \& Davis, G. A. (2004). College student wellness: A comparison between traditional- and nontraditional-age students. Journal of College Counseling, 7, 32 - 39.

Huang, C. L., \& Sumpio, B. E. (2008). Olive oil, the Mediterranean diet, and cardiovascular health. Journal of the American College of Surgeons, 207(3), 407-416.

Issa, C., Darmon, N., Salameh, P., Maillot, M., Batal, M., \& Lairon, D. (2011). A Mediterranean diet pattern with low consumption of liquid sweets and refined cereals is negatively associated with adiposity in adults from rural Lebanon. International Journal of Obesity, 35(2), 251-258.

Papadaki, A., \& Scott, J. A. (2005). The Mediterranean eating in Scotland experience project: Evaluation of an internet-based intervention promoting the Mediterranean diet. The British Journal of Nutrition, 94, 290-298. 
Sahingoz, S. A., \& Sanlier, N. (2011). Compliance with Mediterranean Diet Quality Index (KIDMED) and nutrition knowledge levels in adolescents: A case study from Turkey. Appetite, 57(1), 272277.

Serra-Majem, L. I., Ribas, L., Garcia, A., Perez-Rodrigo, C., \& Aranceta, J. (2003). Nutrient adequacy and Mediterranean diet in Spanish school children and adolescents. European Journal of Clinical Nutrition, 57, 35-39.

Sparling, P. B. (2007). Obesity on campus. Preventing Chronic Disease, 4(3), A72.

Thompson, J. J., \& Porto, S. C. (2014). Supporting wellness in adult online education. International Council for Open and Distance Education, 6(1), 17-28.

Tourlouki, E., Polychronopoulos, E., Zeimbekis, A., Tsakountakis, N., Bountziouka, V., Lioliou, E.,... Panagiotakos, D. B. (2010). The 'secrets' of the long livers in Mediterranean islands: The MEDIS study. European Journal of Public Health, 20, 659-664.

United States Department of Health and Human Services. (2005). Department of Agriculture: Dietary guidelines for Americans. Washington, DC: US Government Printing Office; 2005.

Author Information

Joshua Bernstein PhD, CHES

A.T. Still University of Health Sciences

Department of Health Education

*Basil H. Aboul-Enein, MSc, MPH, MA, DHEd

University of South Dakota

School of Health Sciences

414 E Clark St.

Vermillion, SD 57069

Basil.AboulEnein@usd.edu

* corresponding author 